Original scientific paper

\title{
KINEMATIC MODEL OF A LOGISTIC TRAIN WITH A DOUBLE ACKERMANN STEERING SYSTEM
}

\author{
Paszkowiak, W.; Bartkowiak, T. \& Pelic, M. \\ Poznan University of Technology, Institute of Mechanical Technology, \\ Pl. Marii Sklodowskiej-Curie 5, 60-965 Poznan, Poland \\ E-Mail: wojciech.z.paszkowiak@doctorate.put.poznan.pl
}

\begin{abstract}
The demand for fast, economic and high load capacity intralogistics transport is a growing trend. One of the recent solution is a logistic train, which consists of a tractor and a certain number of towed trailers. It is essential that the trailer follows the track of the preceding unit as it allows reduction of shopfloor area dedicated for transport. A perfectly suited steering system for this application is the double Ackermann system. In this study, the kinematic model of the tractor with $n$ trailers was developed based on the Jacobian matrix. The tractor model was adapted to drive along a given trajectory using a PD controller. The simulation studies confirmed that the PD controller is sufficient to control the trajectory for developed kinematic model. The obtained results showed that the trailers with the double Ackermann steering system follow a similar path, which is the most convenient for narrow turn ride. The developed kinematic model can be used for the fast path planning and the design of transportation routes in manufacturing areas. The expanded model with an advanced control system can be used to control a real logistic train.

(Received in November 2020, accepted in February 2021. This paper was with the authors 2 weeks for 1 revision.)
\end{abstract}

Key Words: Logistic Train, Milk-Run, Kinematic Model, Double Ackermann, Tractor-Trailer System, Trajectory Control

\section{INTRODUCTION}

There is a constant striving in the industry for reduction of production costs and improving the overall efficiency. This also concerns the internal logistics in which new trends can be observed. One of the recent change is the shift from forklift-based transport to so called "milk-run delivery" system [1]. This concept is aimed at limiting the number of intralogistics operations in the manufacturing area. This allows companies to minimize transportation costs and production time [2-4]. The idea of the milk-run stems directly from the method of delivering and receiving supplies in the dairy industry. In this approach, one tanker collects milk with one truck from many dairy farms [5]. It is similar in the industry, but the truck is replaced by the logistic train and dairy farms are simply multiple workstations [6]. Each milk-run route is fixed with a certain sequence of stops serving at least one supplier and being executed cyclically or according to a schedule [7]. In the traditional intralogistics transport many vehicles (e.g. forklifts) are used [8]. Milk-run ensures that one train is sufficient to transport materials between workstations instead of many direct suppliers assigned to each station [9]. A single logistic train during a single round collects and delivers parts or products [10]. The advantage of a logistic train is that it transports more goods than other intralogistics means of transport for each run [11]. One of the main problems with the application of logistics train that the transportation routes were usually designed for single vehicles operating and, hence, have limited dimensions, which makes it difficult for trains to pass without collision with the infrastructure [1]. For this reason, research is currently being conducted into the trajectory analysis of steering systems $[12,13]$.

A logistic train consist of a tractor and a certain number of towed trailers. The tractor towing a set of trailers usually has a classic Ackermann steering system or a rear-wheel differential drive [12-15]. Ackermann steering system involves one or two steering wheels at the front. A 
tractor with a differential drive is similar to a wheeled mobile robot (WMR), where the two rear wheels are driven independently $[14,16]$. The front passive wheel simply adapts to the direction of movement. The difference of rotational speeds (assuming that the rear wheels have the same diameter) between the rear wheels causes the tractor to turn [14, 17].

Trailer steering systems concern two aspects, namely, the way of connecting trailers to adjacent trailers and a tractor, as well as the types of wheels and axles. The conventional steering systems have proved insufficient for trains with a large number of trailers $[12,13]$. It contains fixed drawbars, two rear fixed wheels and two caster wheels at the front or only two rear fixed wheels [18]. The double Ackermann is a more advanced steering system. It has a rotary drawbar at the front and four swivel wheels, the turning of which is coupled with the rotation of that drawbar. The turn of the front rotary drawbar determines the turn of the trailer wheels according to the Ackermann principle. The turning direction of the front wheels is the same as the front drawbar and opposite for the rear wheels. An illustrative comparison between the two discussed solutions is shown in Fig. 1 [12, 13].

a)

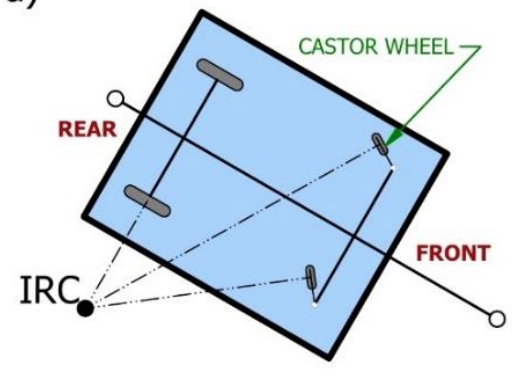

b)

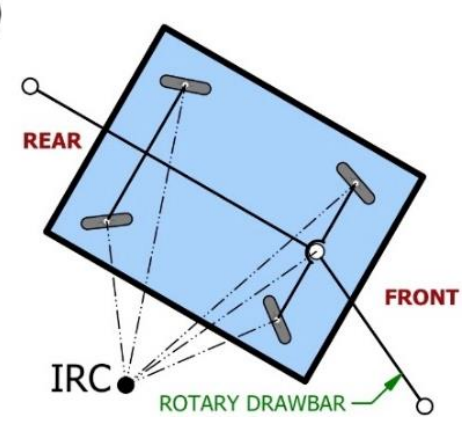

Figure 1: A comparison of steering systems for logistic trailers: a) conventional steering system, b) double Ackermann steering system (IRC - instant centre of rotation).

Kinematic modelling of a logistic train can be treated as modelling of an articulated $n$-trailer vehicle [19]. These vehicles are considered with dynamic models, where the kinematic model is part of the dynamic modelling of the nonholonomic system [20, 21]. Nonholonomic vehicle models have constraints to ensure no slip ride. These non-slip conditions are described for each wheel. Velocity is assumed to be zero in the lateral direction for each wheel [22]. The kinematic dependencies are described in the Jacobian matrix [23]. This matrix is related to the matrix of system constraint. The result of the multiplication of these two matrices is zero [24]. The nonholonomic dynamic model is similar to the kinematic model [25]. Such a dynamic model is more advanced and includes masses, moments of inertia and a driving force [26]. In case of non-aggressive driving, the trajectory of the train may be sufficiently described and simulated via kinematic model [27]. The kinematic model of the logistic train was considered by Paszkowiak et al. [13]. Three types of steering systems were compared including the double Ackermann steering system. The proposed model has been validated for real system using trajectory captured by means of aerial drone. This kinematic model was based only on position analysis and the calculation were performed iteratively. The results obtained in the previous iteration were used for the subsequent step. The accuracy of the motion can be controlled by the refinement of iteration. The movement of the train in a particular iteration was divided into individual stages. Each iteration started with the movement of the tractor. This was followed by motion of the trailers, one by one, from the first to the last. Each stage of moment involves rotation and translation to maintain connection between adjacent vehicles. For the double Ackermann steering system, the rotation of the trailer was performed relative to its centre, and the rotation of the rotary drawbar with respect to its connection to the trailer. It can be seen that this method is quite complicated and very laborious. The velocities were not even taken into account as input values. In this model, the time parameter is not directly represented. One of 
the weaknesses of this model is the difficulty in the application of the control system. This makes it impossible to calculate a specific trajectory [13].

The objective of this paper is to present a kinematic model of a logistic train consisting of trailers with double Ackermann steering system. The model is used here for trajectory control. The proposed calculation procedure is not complicated and the computation time is relatively fast. The dynamics of the train is not included in the model for several reasons. The first reason is to get the computation time as short as possible, the second is to obtain a simplified calculation procedure that does not require many irrelevant parameters. The last one is that there no need to take into account the dynamic phenomena during the non-aggressive running of the logistic train.

In this paper, a new kinematic model of the modern double Ackermann steering system for internal transport based on Milk-Run concept is presented. The development of this model based on the Jacobian matrix enabled the implementation of a trajectory control system. The double Ackermann steering has never been used in wheeled robots or similar multiple traileron-tractor systems. It provides a great advantage of trajectory maintenance for all towed trailers so a drive through narrow and windy corridors can be potentially possible when compared to other steering system. However, an application of the double Ackermann system creates an additional challenge in the modelling of the system dynamics. In order to reduce this complexity, a kinematic model was developed and described in this paper. The main benefit of the proposed approach is the reduction of calculation time when compared to the dynamic model involving frictional effects. We believe the hereby described model can be accurate for non-aggressive driving style which applies to most industrial applications of logistic trains and wheeled robots. Based on the literature review the double Ackermann steering system has not yet been considered for automated guided applications. Therefore, a simple and easy-toimplement kinematic model for this steering system can provide a better understanding of the system behaviour and its control.

\section{KINEMATIC MODEL}

The purpose of this section is to demonstrate how the kinematic model for a logistic train including tractor with $n$ trailers is designed. A graphical representation of the entire train is presented in Fig. 2.

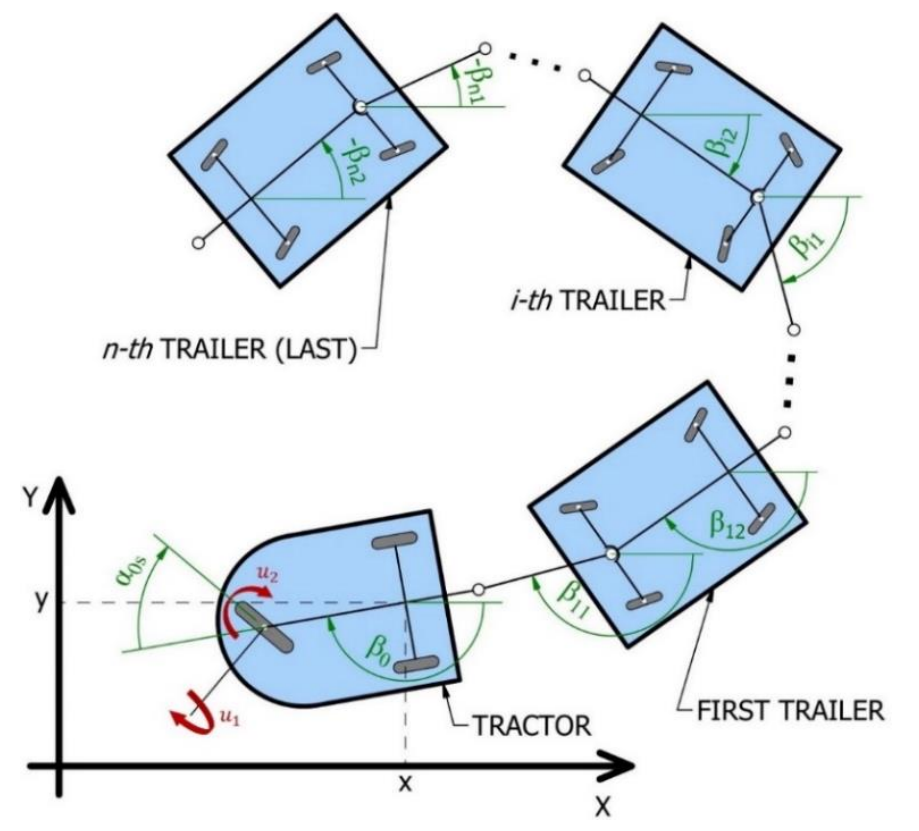

Figure 2: The tractor with $n$ trailers and the generalized coordinates. 
The tractor is a described using tricycle model [28]. The wheel at the front is steered and driven. Two passive rear wheels are fixed. The input parameters are the angular velocity of wheel rotation and the angular velocity of the turning wheel. Both concern the front wheel of the tractor. The position of the tractor can be described based on four generalized coordinates. Two of them determine the location of the characteristic point of the tractor $\mathrm{B}_{0}$ (see Fig. $3 \mathrm{a}$ ). The next coordinate constitutes the angular position on the plane. This is $\beta_{0}$ angle, which is measured between the main axis of the tractor and the horizontal line. The last generalized coordinate of the tractor is the angle describing the angular position of the front wheel in relation to the main axis of the tractor.

The trailers with double Ackermann steering system are divided into two components. The first one is the rotary drawbar, and the second is the body of the trailer (see Fig. $3 \mathrm{~b}$ ). Each of these elements has one generalized coordinate. For the $i^{\text {th }}$ rotary drawbar it is $\beta_{i 1}$ angle, and for the $i^{\text {th }}$ trailer $-\beta_{i 2}$ angle. Both describe the angular positions of drawbar/trailer relative to the horizontal line. The angles describing the angular position of the trailer wheels are not included in the generalized coordinate vector, because they are not needed to calculate the trajectory of the trailers. These angles are closely related to the angular angles of the trailer and the drawbar (see Fig. $3 \mathrm{c}$ ). Therefore, the vector of generalized coordinates for the entire system (tractor and $n$ trailers) can be represented as:

$$
\mathbf{q}=\left[\begin{array}{llllllllllll}
x & y & \beta_{0} & \alpha_{0 s} & \beta_{11} & \beta_{12} & \cdots & \beta_{i 1} & \beta_{i 2} & \cdots & \beta_{n 1} & \beta_{n 2}
\end{array}\right]^{T}
$$

a)

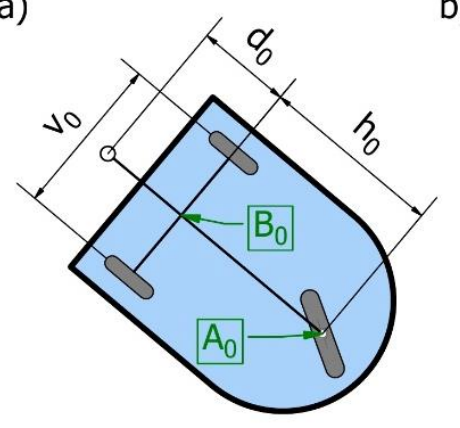

b)

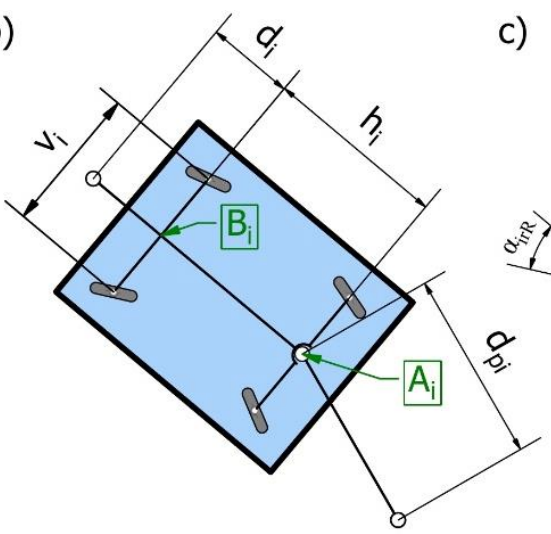

c)

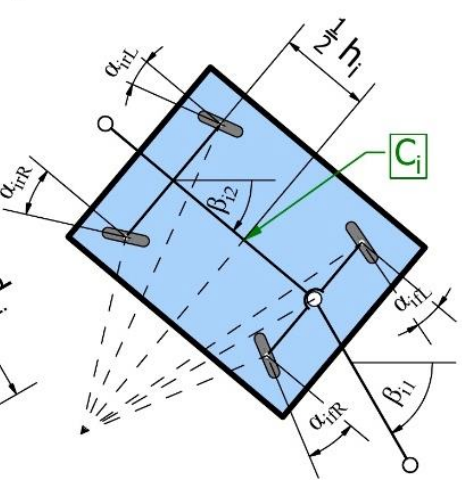

Figure 3: Basic parameters; a) tractor as a tricycle model, b) $i^{\text {th }}$ trailer with double Ackermann steering system, c) steering angles for $i^{\text {th }}$ trailer.

All generalized coordinates are time-dependent. The main equation of the kinematic model for the given system can be written as:

$$
\dot{\mathbf{q}}=\mathbf{J}(\mathbf{q}) \mathbf{u}
$$

where $\mathbf{J}(\mathbf{q})$ is the Jacobian matrix and $\mathbf{u}$ is a $2 \times 1$ velocity vector containing the input parameters. This vector is given as:

$$
\mathbf{u}=\left[\begin{array}{ll}
u_{1} & u_{2}
\end{array}\right]^{T}
$$

where $u_{1}$ is the rotational velocity of the front wheel, and $u_{2}$ is the angular velocity of the turn of the front wheel $\dot{\alpha}_{0 s}$, both referring to the tractor. The Jacobian matrix describes the generalized velocities based on the input velocities included in $\mathbf{u}$. Therefore, this matrix is the relationship between the input parameters and the generalized velocities. In order to create the matrix, the velocity vectors were analysed. To obtain the velocity of the trailers, it is essential to calculate the velocities of the tractor body. The velocities include the linear velocity of $\mathrm{B}_{0}$ point and corresponding rotational velocity. These velocities have to be obtained using the input vector $\mathbf{u}$. The relationships between the input parameters and the linear $\left(f_{l 0}\right) /$ rotational $\left(f_{a 0}\right)$ velocity in the local coordinate system of the tractor can be written as: 


$$
\begin{aligned}
& f_{l 0}=r_{0 f} \cos \left(\alpha_{0 s}\right) \\
& f_{a 0}=\frac{r_{0 f}}{h_{0}} \sin \left(\alpha_{0 s}\right)
\end{aligned}
$$

where $r_{0 f}$ is a radius of the front wheel of the tractor. Inside the Jacobian matrix, these relationships must be formulated relative to the global coordinate system. Therefore, the Jacobian matrix for the discussed system with $n$ trailers is matrix of $(4+2 n) \times 2$ size. Each trailer takes two rows, the tractor takes four. The two columns result from the two-element input vector. For this system, the Jacobian matrix can be defined as:

$$
\mathbf{J}(\mathbf{q})=\left[\begin{array}{cccccccccccc}
f_{l 0} \cos \left(\beta_{0}\right) & -f_{l 0} \sin \left(\beta_{0}\right) & f_{a 0} & 0 & f_{a 11} & f_{a 12} & \cdots & f_{a i 1} & f_{a i 2} & \cdots & f_{a n 1} & f_{a n 2} \\
0 & 0 & 0 & 1 & 0 & 0 & \cdots & 0 & 0 & \cdots & 0 & 0
\end{array}\right]^{T}
$$

For the rotary drawbar of the first trailer, the relationships for linear (subscript $l$ )/angular (subscript $a$ ) velocity can be described as:

$$
\begin{aligned}
& f_{l 11}=f_{l 0} \cos \left(\beta_{0}-\beta_{11}\right)+f_{a 0} \cdot d_{0} \sin \left(\beta_{0}-\beta_{11}\right) \\
& f_{a 11}=\frac{f_{l 0} \sin \left(\beta_{0}-\beta_{11}\right)-f_{a 0} \cdot d_{0} \cos \left(\beta_{0}-\beta_{11}\right)}{\mathrm{d}_{\mathrm{p} 1}}
\end{aligned}
$$

These velocities refer to the connection point of the rotary drawbar with the trailer to which this drawbar belongs. For the first trailer, the velocity relationships can be described similarly as for the $i^{\text {th }}$ trailer. The relationships for the rotary drawbar of the $i^{\text {th }}$ trailer (subscript $i 1$ ) and itself (subscript $i 2$ ) can be written as:

$$
\begin{gathered}
f_{l i 1}=f_{l(i-1) 2} \cos \left(\beta_{(i-1) 2}-\beta_{i 1}\right)+f_{a(i-1) 2} \cdot\left(d_{i-1}+\frac{h_{i-1}}{2}\right) \sin \left(\beta_{(i-1) 2}-\beta_{i 1}\right) \\
f_{a i 1}=\frac{f_{l(i-1) 2} \sin \left(\beta_{(i-1) 2}-\beta_{i 1}\right)+f_{a(i-1) 2} \cdot\left(d_{i-1}+\frac{h_{i-1}}{2}\right) \cos \left(\beta_{(i-1) 2}-\beta_{i 1}\right)}{\mathrm{d}_{\mathrm{pi}}} \\
f_{l i 2}=f_{l i 1} \cos \left(\beta_{i 1}-\beta_{i 2}\right) \\
f_{a i 2}=\frac{2 f_{l i 1}}{\mathrm{~h}_{\mathrm{i}}} \sin \left(\beta_{i 1}-\beta_{i 2}\right)
\end{gathered}
$$

The main equation of the system can be solved numerically to obtain the interpolating functions of the generalized coordinates. Then, the logistic train can be visualized in time. At this stage, it is necessary to determine the steering angles in the trailers with the double Ackermann steering system. These angles for the $i^{\text {th }}$ trailer are shown in Fig. $3 \mathrm{c}$ and can be denoted as:

$$
\left\{\begin{array}{c}
\alpha_{i f L}=\operatorname{arctg}\left(\frac{h_{i}}{v_{i}+h_{i} \operatorname{ctg}\left(\beta_{i 1}-\beta_{i 2}\right)}\right) \\
\alpha_{i f R}=\operatorname{arctg}\left(\frac{h_{i}}{-v_{i}+h_{i} \operatorname{ctg}\left(\beta_{i 1}-\beta_{i 2}\right)}\right) \\
\alpha_{i r L}=-\alpha_{i f L} \\
\alpha_{i r R}=-\alpha_{i f R}
\end{array}\right.
$$

where, the subscripts: $f, r, L, R$ refer to front, rear, left, right respectively. The steering angles of the wheels are measured in the same direction. This causes the rotation of rear wheels to have the opposite sign to the front ones.

\section{DESIGN OF KINEMATIC CONTROLLER}

In this section the kinematic controller is presented for trajectory control that can allow the tractor to drive along a given path. The specific values can be given as input parameters in $\mathbf{u}$, 
see Eq. (3). However, it is reasonable to provide a specific trajectory to guide the train. For this purpose, a PD controller was used. In that case, the input parameters can be described as:

$$
\begin{gathered}
u_{1}=\left\{\begin{array}{cc}
K_{p 1} e_{p}+K_{v 1} \dot{e}_{p} & K_{p 1} e_{p}+K_{v 1} \dot{e}_{p}<\theta_{\max } / r_{0 f} \\
\theta_{\max } / r_{0 f} & K_{p 1} e_{p}+K_{v 1} \dot{e}_{p} \geq \theta_{\max } / r_{0 f}
\end{array}\right. \\
u_{2}=\left\{\begin{array}{cc}
-\omega_{\max } & -\omega_{\max } \geq K_{p 2} e_{\alpha}+K_{v 2} \dot{e}_{\alpha} \\
K_{p 2} e_{\alpha}+K_{v 2} \dot{e}_{\alpha} & -\omega_{\max }<K_{p 2} e_{\alpha}+K_{v 2} \dot{e}_{\alpha}<\omega_{\max } \\
\omega_{\max } & K_{p 2} e_{\alpha}+K_{v 2} \dot{e}_{\alpha} \geq \omega_{\max }
\end{array}\right.
\end{gathered}
$$

where $K_{p j}$ and $K_{v j}$ are the proposed controller gains, $\theta_{\max }$ is a maximum velocity of the tractor (point $\mathrm{A}_{0}$ ), $\omega_{\max }$ is a maximum torsional velocity of the front wheel of the tractor, $e_{p}$ is a position error (relative to the point $\mathrm{A}_{0}$ ), $e_{\alpha}$ is an orientation error for the front wheel of the tractor. These errors are showed in Fig. 4 and can be defined as:

$$
\left\{\begin{array}{c}
e_{p}=\sqrt{e_{x}^{2}+e_{y}^{2}} \\
e_{\alpha}=e_{\beta}-\beta_{0}-\alpha_{0 s}=\operatorname{arctg}\left(\frac{-e_{y}}{e_{x}}\right)-\beta_{0}-\alpha_{0 s}
\end{array}\right.
$$

where the arctg function used above is a special function commonly known as arctg2, where numerator and denominator can be distinguished. This allows obtaining an angle within the range from $-\pi$ to $\pi$. Position error components $\left(e_{x}, e_{y}\right)$ are given as:

$$
\left\{\begin{array}{l}
e_{x}=x_{A 0 d}-x_{A 0}=x_{A d}-x-h_{0} \cos \left(\beta_{0}\right) \\
e_{y}=y_{A 0 d}-y_{A 0}=\mathrm{y}_{A d}-y+h_{0} \sin \left(\beta_{0}\right)
\end{array}\right.
$$

where $x_{A 0 d}, y_{A 0 d}$ are the coordinates of a point moving along the reference path, $x_{A 0}, y_{A 0}$ are the coordinates of point $\mathrm{A}_{0}$.

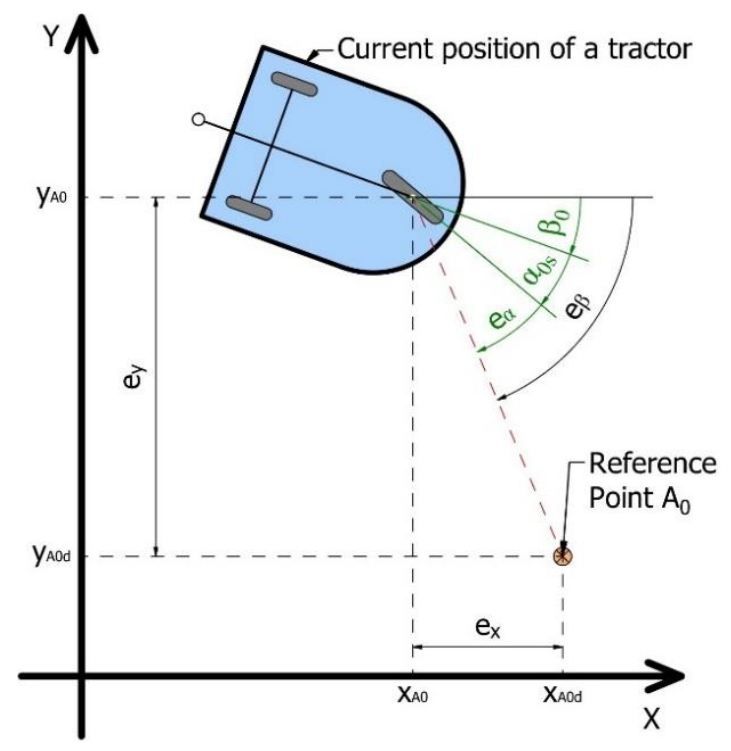

Figure 4: Current position of a tractor and errors relative to the $\mathrm{A}_{0}$ reference point.

\section{SIMULATION}

The developed simulation system includes a tractor as a tricycle model, which is common for a logistic tractor and four trailers with the double Ackermann steering system. The selected number of trolleys is common in logistic trains, and both enable clear visualization in a small space. Simulations have been performed in Mathematica 12 (Wolfram, UK). The reference path is assumed for the tractor $\mathrm{A}_{0}$ point. The controller gains of the proposed controller are selected by trial and error as: 


$$
K_{p 1}=100, K_{v 1}=50, K_{p 2}=500, K_{v 2}=50
$$

The geometric properties of the system are given in Table I. These geometric parameters are assumed for the logistic reference train. For simulation, it was assumed that the maximum velocity of the tractor $\theta_{\max }$ is $50 / 9 \mathrm{~m} / \mathrm{s}(20 \mathrm{~km} / \mathrm{h}$ - the most common maximum velocity of a logistic tractor) and maximum torsional velocity of the front wheel of the tractor $\omega_{\max }$ is $\pi \mathrm{rad} / \mathrm{s}$. The simulations were performed for two different trajectories. The logistic train running time in the simulation was 20 seconds. First one is a circle, and the latter is a shape of the eight. For first scenario, trajectory is given as:

$$
x_{A 0 d}=3 \cos \left(\frac{\pi t}{10}\right), y_{A 0 d}=3 \sin \left(\frac{\pi t}{10}\right)
$$

and for the second scenario as:

$$
x_{A 0 d}=5 \sin \left(\frac{\pi t}{10}\right), y_{A 0 d}=2.5 \sin \left(\frac{\pi t}{5}\right)
$$

The initial conditions are given according to the generalized coordinates, see Eq. (1), as:

$$
\mathbf{q}(0)=\left[\begin{array}{llllllllllll}
5 & -5 & 0 & 0 & 0 & 0 & 0 & 0 & 0 & 0 & 0 & 0
\end{array}\right]^{T}
$$

Table I: Geometric properties of the system.

\begin{tabular}{|c|c|}
\hline Parameter & Value (m) \\
\hline $\boldsymbol{h}_{\mathbf{0}}$ & 1 \\
\hline $\boldsymbol{v}_{\mathbf{0}}$ & 0.5 \\
\hline $\boldsymbol{r}_{\mathbf{0} \boldsymbol{f}}$ & 0.2 \\
\hline $\boldsymbol{d}_{\mathbf{0}}, \boldsymbol{d}_{\boldsymbol{i}}$ & 0.2 \\
\hline $\boldsymbol{h}_{\boldsymbol{i}}$ & 0.8 \\
\hline $\boldsymbol{d}_{\boldsymbol{p} \boldsymbol{i}}$ & 0.4 \\
\hline $\boldsymbol{v}_{\boldsymbol{i}}$ & 0.3 \\
\hline
\end{tabular}

\subsection{Scenario 1}

Fig. 5 shows the tracking errors. In the initial stage, the position error increases (see Fig. 5 a). This is due to the position and orientation controllers work simultaneously and limit the angular velocity of wheel turning. As a result, the turning of the wheel did not have enough time to reach the proper orientation, while the train was on its way.

a) $e_{p}[\mathrm{~m}]$

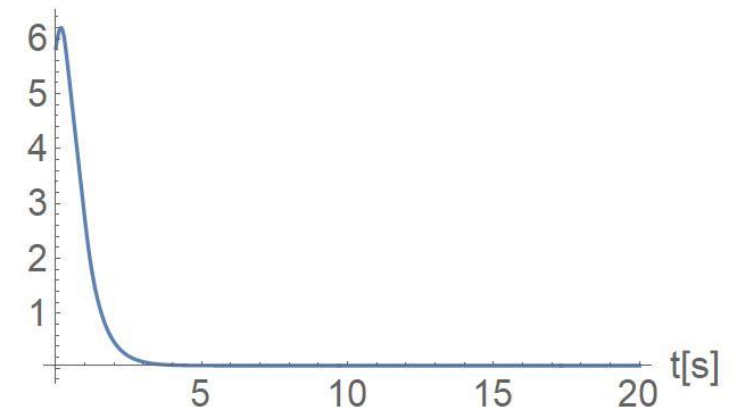

b)

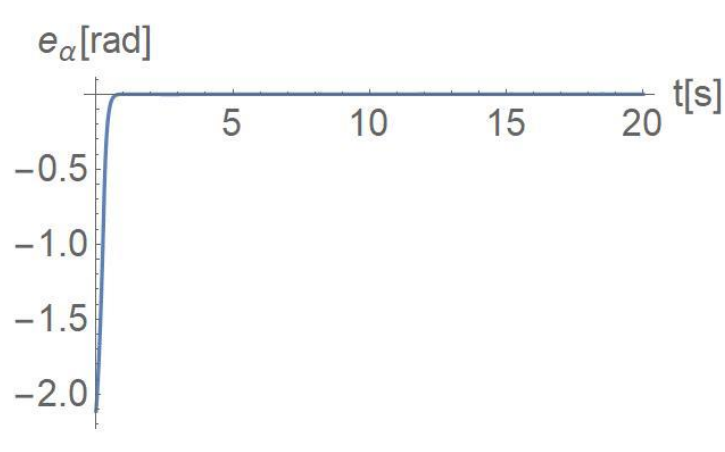

Figure 5: Tracking errors, a) position and b) orientation error plotted as a function of time.

The orientation error tends to zero from the start of simulation (see Fig. 5 b). In Fig. 6 a, the given and simulation trajectory of the tractor point $\mathrm{A}_{0}$ is presented. There is shown that the tractor maintains its desired trajectory after reaching the reference point. 

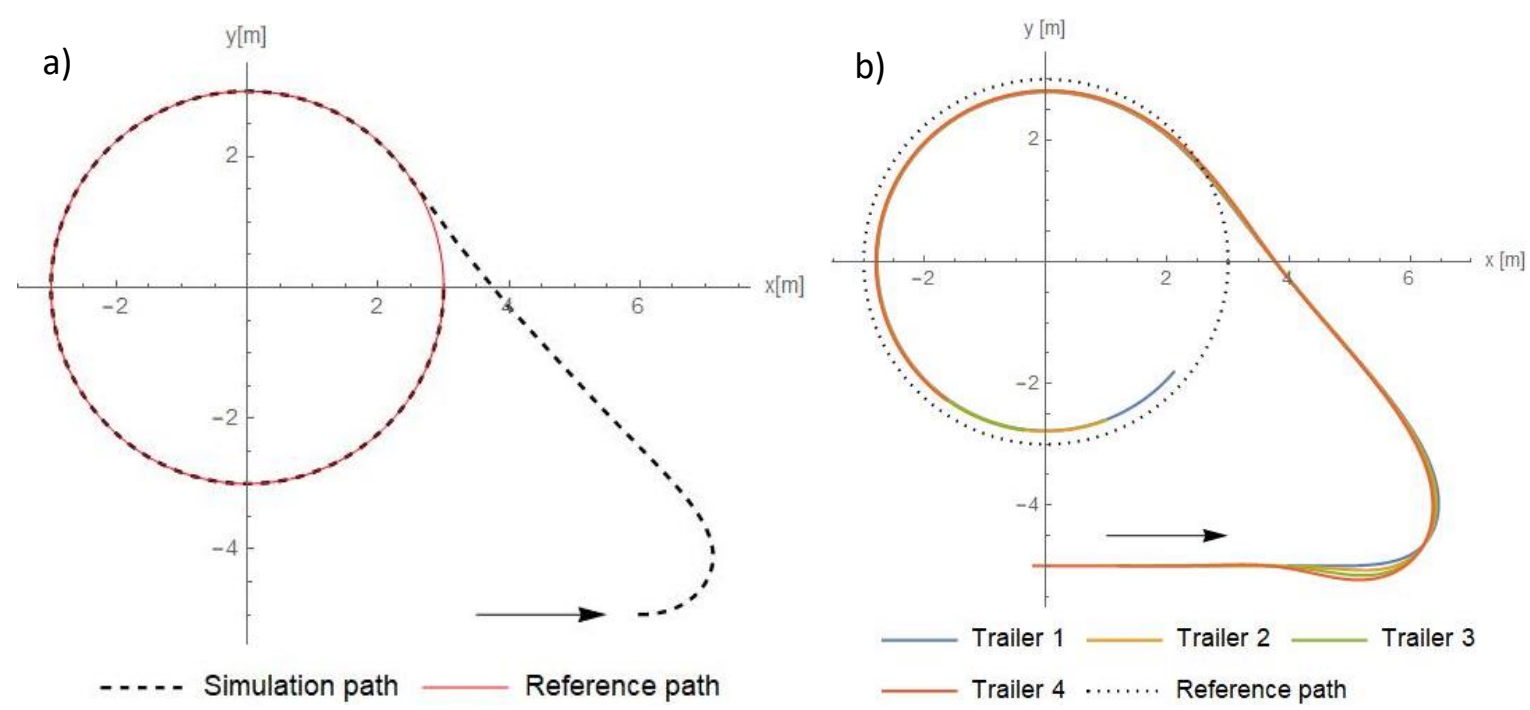

Figure 6: Reference and simulation path: a) for a point $\mathrm{A}_{0}$ of the tractor, $\mathrm{b}$ ) of the point $\mathrm{C}_{i}$ for each trailer (the arrow visualizes the initial direction of motion).

The main purpose of the article is to analyse the trajectory of trailers as they are objects which can potentially collide with the shopfloor infrastructure or personnel. The position of the centre of each of the trailer is presented in Fig. 6 b. There is shown that the trailers with double Ackermann steering system obtain very similar trajectories. The path discrepancies are noticeable only in the case of a sharp turn that occurred at the beginning of the run. It is obvious that the trailer centre is not capable of following the trajectory set for the centre of the front wheel of the tractor during cornering. This is due to the different steering system between the tractor and the trailers.

The deviation between this trajectory and the trailer centres is presented in Fig. 7. There is also noticeable similarity of trajectories. From visual impression, a great similarity between trailers trajectories can be noted. The shape of curve is noticeably different for the first trailer. This is because the unit in front of it is not a trailer but a tractor with a different steering system. The values stabilized at a very similar value for all trailers.

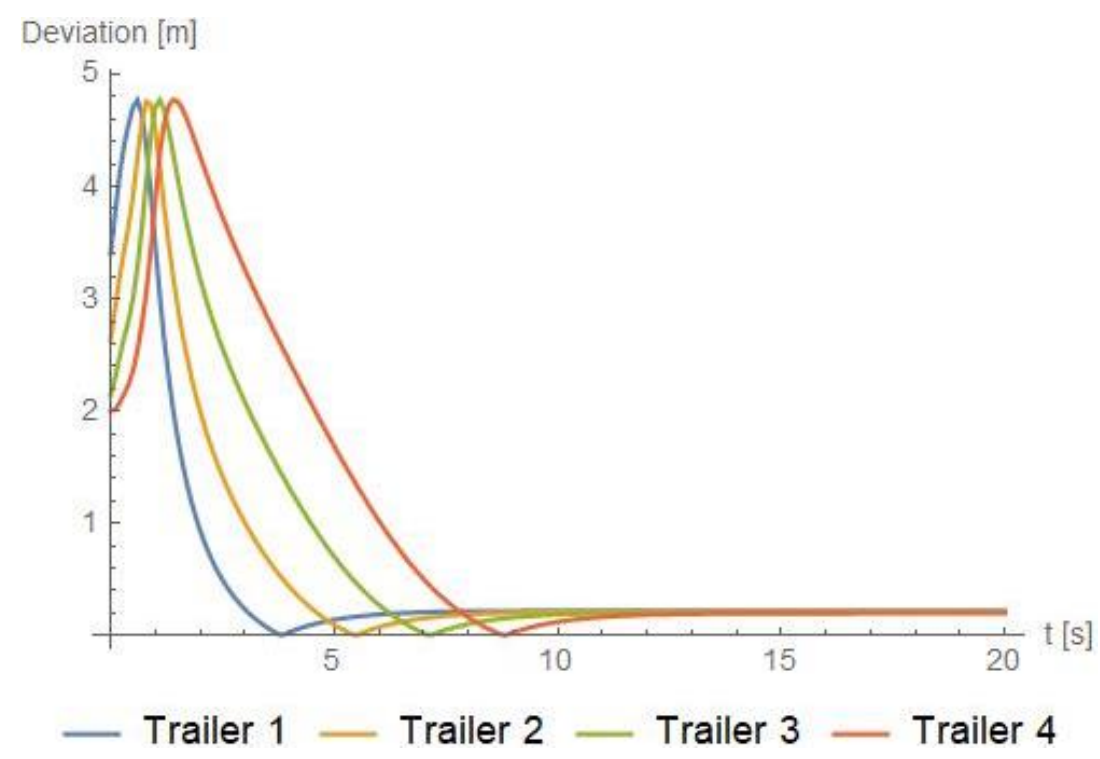

Figure 7: The closest distance between the point $\mathrm{C}_{i}$ and reference path.

In order to visualize the train during its run, the train is rendered for selected time frames as showed in Fig. 8. This figure visualized a potential cause for the discrepancies in paths as 
presented earlier in Fig. 7. Point $\mathrm{B}_{0}$ cannot follow the reference path during a turn. This is due to the distance $h_{0}$ between the front wheel and the rear axle of the tractor (see Fig. 3 a).

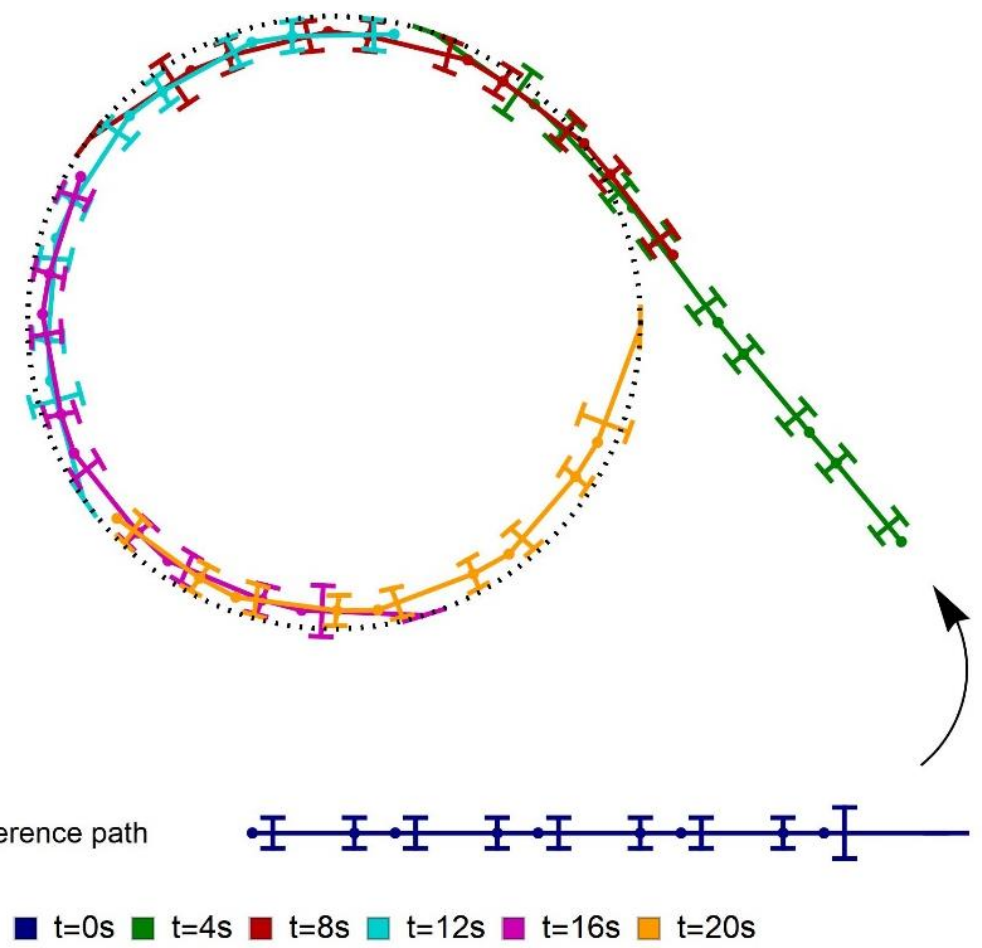

Figure 8: The visualization of the train for selected time frames (the arrow indicates the initial direction of motion).

\subsection{Scenario 2}

In Fig. 9, tracking errors are presented. The waveforms are similar to the previous one. In the initial stage the position error increases in the same way as previous. In Fig. 10 a, the given and simulation trajectory of the point $\mathrm{A}_{0}$ are shown. Despite the more complicated shape of the trajectory than in the previous scenario, the tractor maintains the given trajectory. The location of the centre of each of the trailers is presented in Fig. $10 \mathrm{~b}$. For the eight shaped the similarity of trajectories is also noticeable, but not as clearly as for the circular trajectory. This is due to more complex shape and sharper manoeuvres.

a) $e_{p}[m]$

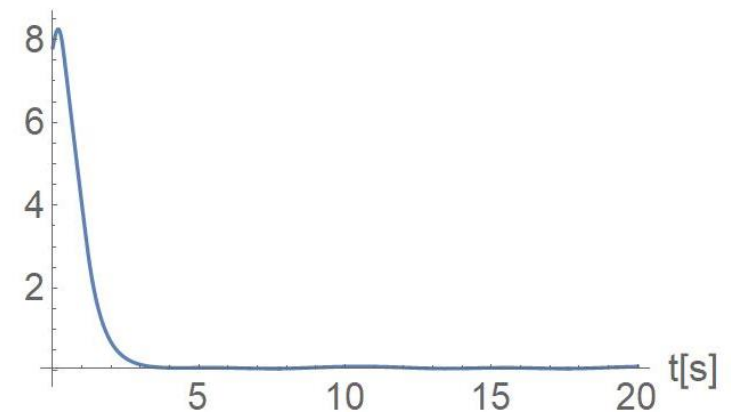

b)

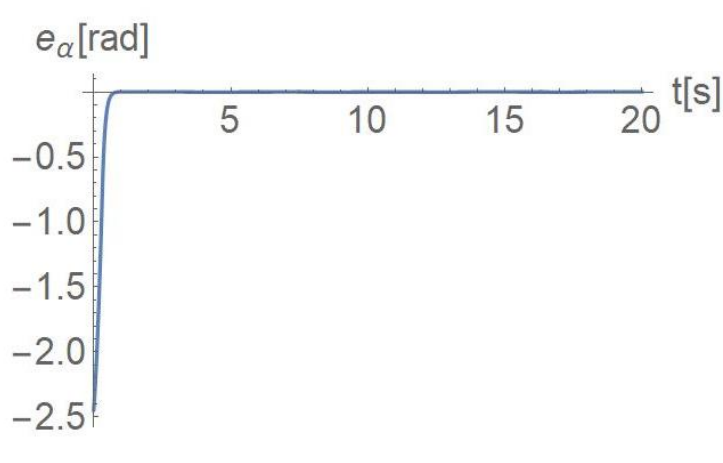

Figure 9: Tracking errors, a) position and b) orientation error plotted as a function of time.

The visualization of the train for selected time frames is shown in Fig. 11. Due to the more complicated shape of the trajectory, the deviation was not calculated. The deviation can be estimated based on Fig. $10 \mathrm{~b}$ and Fig. 11. Fig. 11 shows that the distance $h_{0}$ causes a greater deviation between the reference path and the trajectories of the trailers for sharper turns. For a 
time equal to $4 \mathrm{~s}$ (green train), the train takes the sharpest turn of the whole route. For this moment, the deviation for each trailer is the greatest.

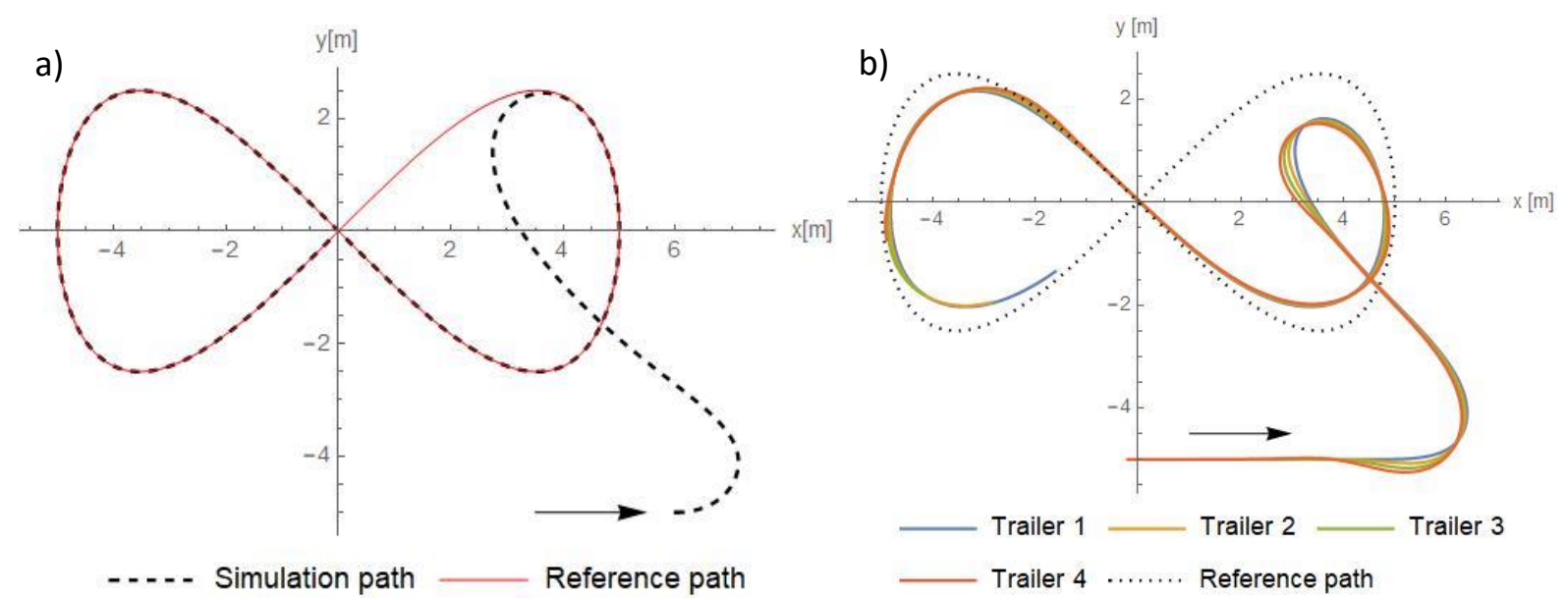

Figure 10: Reference and simulation path: a) for a point $\mathrm{A}_{0}$ of the tractor, b) of the point $\mathrm{C}_{i}$ for each trailer.
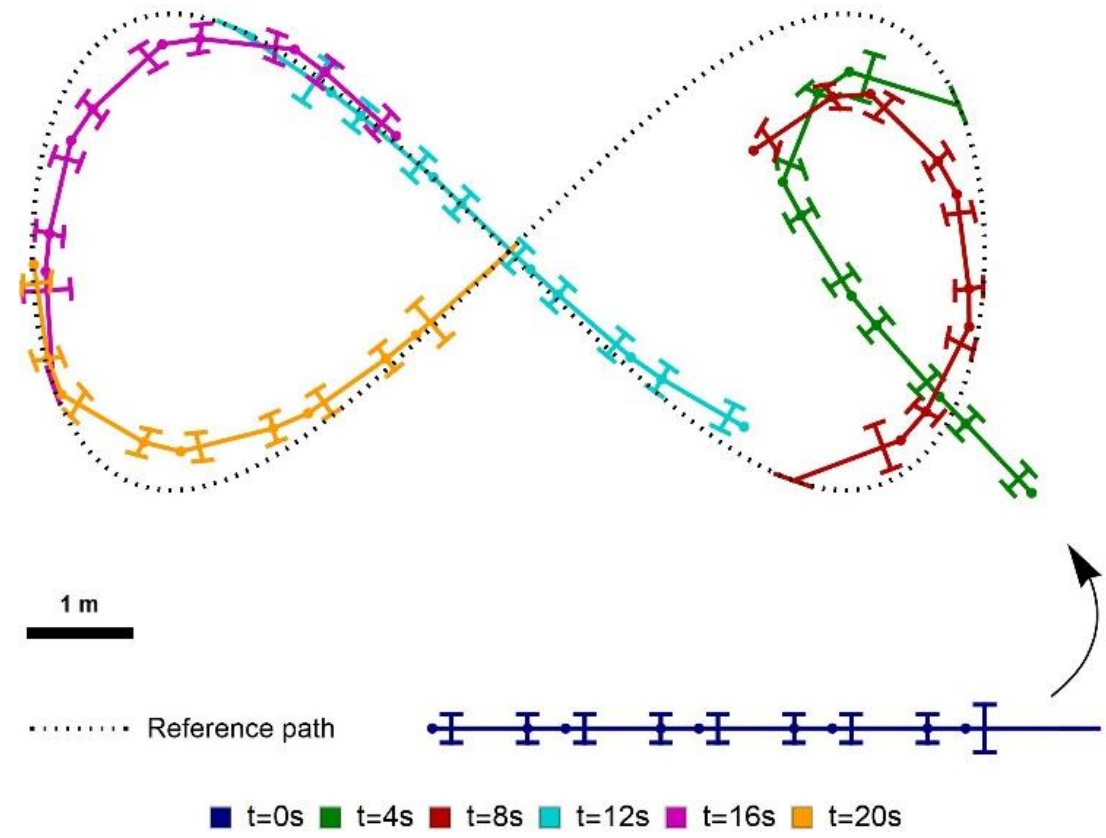

Figure 11: The visualization of the train for selected moments in time.

\section{CONCLUSION}

Kinematic modelling of the logistic train with $n$ trailers with the double Ackermann steering system for application with rides along a given trajectory was presented in this paper. Kinematic model was based on the Jacobian matrix. In order to track the position of the train a PD controller used, which was based on position and orientation errors. These errors were calculated according to a reference point that moved in time. In the initial test, PID controller was tested and no significant improvement was noted. The mere proportional controller itself should ensure that the desired position is reached, but it cannot slow down sufficiently before reaching the target position.

The kinematics of the double Ackermann steering system was analysed. The velocity impact was not taken into account because geometrical parameters are sufficient to obtain the trajectory only on the basis of kinematics. Dynamic parameters were not included as well, 
because no slip and the shortest computation time were assumed. The obtained results showed that the trajectories of the trailer centre were similar. The centre of the trailers is not too close to the path of the reference point. Using a different type of a drive, especially a differential drive may solve this problem. In the case of a rear differential drive, the given trajectory should be the reference path of the point $\mathrm{B}_{0}$ (centre of the rear axle). Changing geometric parameters can also increase the similarity of covered trajectories. Merely changing the reference point to $\mathrm{B}_{0}$ and modifying the controller may also reduce the distance between the centre of the trailers and the reference path. It is obvious that increasing the geometric parameters of the system will require wider transportation roads. On the other hand, reducing them, especially the length of the drawbars, may cause a collision between the trailers. It also reduces the manoeuvrability of the train. Therefore, a balance between those two factors needs to be found.

The presented kinematic model can be surely used to analyse the trajectory of a logistic train with a double Ackermann steering system. It allows determining whether the train is able to pass the given route without a collision with shopfloor infrastructure. The developed model provides a shorter computation time than dynamic models or a kinematic model with an iterative calculation procedure. The expanded model with an advanced control system can be used to control a real logistic train, and even an autonomous system.

\section{ACKNOWLEDGEMENT}

This project was supported by the Polish Ministry of Science and Higher Education as a part of annual subsidy, project number: 0614/SBAD/1529.

\section{REFERENCES}

[1] Satoh, I. (2008). A formal approach for milk-run transport logistics, IEICE Transactions on Fundamentals of Electronics, Communications and Computer Sciences, Vol. 91, No. 11, 32613268, doi:10.1093/ietfec/e91-a.11.3261

[2] Bocewicz, G.; Banaszak, Z.; Rudnik, K.; Witczak, M.; Smutnicki, C.; Wikarek, J. (2020). Milkrun routing and scheduling subject to fuzzy pickup and delivery time constraints: an ordered fuzzy numbers approach, Proceedings of the 2020 IEEE International Conference on Fuzzy Systems, 10 pages, doi:10.1109/FUZZ48607.2020.9177733

[3] Kluska, K. (2019). Yamazumi analysis in milk-run intralogistics systems using simulation tools, Burduk, A.; Chlebus, E.; Nowakowski, T.; Tubis, A. (Eds.), Intelligent Systems in Production Engineering and Maintenance, Springer, Cham, 509-519, doi:10.1007/978-3-319-97490-3_49

[4] Kilic, H. S.; Durmusoglu, M. B.; Baskak, M. (2012). Classification and modeling for in-plant milkrun distribution systems, The International Journal of Advanced Manufacturing Technology, Vol. 62, No. 9-12, 1135-1146, doi:10.1007/s00170-011-3875-4

[5] Kluska, K.; Pawlewski, P. (2018). The use of simulation in the design of milk-run intralogistics systems, IFAC-PapersOnLine, Vol. 51, No. 11, 1428-1433, doi:10.1016/j.ifacol.2018.08.314

[6] Lieb, C.; Prinz, T.; Gunthner, W. A.; Fottner, J. (2019). Modeling and simulation of generic handling operations in in-plant milk-run systems, Proceedings of the $8^{\text {th }}$ International Conference on Industrial Technology and Management, 218-223, doi:10.1109/icitm.2019.8710720

[7] Meyer, A. (2017). Milk Run Design: Definitions, Concepts and Solution Approaches, KIT Scientific Publishing, Karlsruhe, doi: $10.5445 / \mathrm{ksp} / 1000057833$

[8] Straka, M.; Khouri, S.; Lenort, R.; Besta, P. (2020). Improvement of logistics in manufacturing system by the use of simulation modelling: A real industrial case study, Advances in Production Engineering \& Management, Vol. 15, No. 1, 18-30, doi:10.14743/apem2020.1.346

[9] Knez, M.; Gajsek, B. (2015). Implementation of in-plant milkrun system for material supply in lean automotive parts manufacturing, Proceedings of the 2015 International Conference on Logistics \& Sustainable Transport, 121-126

[10] Mao, Z.; Huang, D.; Fang, K.; Wang, C.; Lu, D. (2019). Milk-run routing problem with progresslane in the collection of automobile parts, Annals of Operations Research, Vol. 291, No. 1-2, 657 684, doi:10.1007/s10479-019-03218-X 
[11] Alnahhal, M.; Ridwan, A.; Noche, B. (2014). In-plant milk run decision problems, Proceedings of the 2014 International Conference on Logistics Operations Management, 85-92, doi:10.1109/ gol.2014.6887421

[12] Paszkowiak, W.; Bartkowiak, T. (2021). Dynamic model of a logistic train with different steering systems and tire models, Latin American Journal of Solids and Structures, Vol. 18, No. 1, Paper e339, 27 pages, doi:10.1590/1679-78256147

[13] Paszkowiak, W.; Bartkowiak, T.; Pelic, M. (2019). Kinematic model of multiple trailers on a tractor system for production logistics applications, Archives of Mechanical Technology and Materials, Vol. 39, No. 1, 16-25, doi:10.2478/amtm-2019-0004

[14] Trojnacki, M.; Dąbek, P. (2019). Mechanical properties of modern wheeled mobile robots, Journal of Automation, Mobile Robotics and Intelligent Systems, Vol. 13, No. 3, 3-13, doi:10.14313/ jamris/3-2019/21

[15] Li, L. (2017). Nonholonomic motion planning using trigonometric switch inputs, International Journal of Simulation Modelling, Vol. 16, No. 1, 176-186, doi:10.2507/IJSIMM16(1)CO5

[16] Muthukumaran, S.; Sivaramakrishnan, R. (2019). Optimal path planning for an autonomous mobile robot using dragonfly algorithm, International Journal of Simulation Modelling, Vol. 18, No. 3, 397-407, doi:10.2507/IJSIMM18(3)474

[17] Staicu, S. (2009). Dynamics equations of a mobile robot provided with caster wheel, Nonlinear Dynamics, Vol. 58, No. 1-2, 237-248, doi:10.1007/s11071-009-9474-3

[18] Šušteršič, G.; Prebil, I.; Ambrož, M. (2014). The snaking stability of passenger cars with light cargo trailers, Strojniski vestnik - Journal of Mechanical Engineering, Vol. 60, No. 9, 539-548, doi: $10.5545 /$ sv-jme.2014.1690

[19] Bravo-Doddoli, A.; García-Naranjo, L. C. (2015). The dynamics of an articulated $n$-trailer vehicle, Regular and Chaotic Dynamics, Vol. 20, No. 5, 497-517, doi:10.1134/s1560354715050019

[20] Chen, C.-C.; Li, J.-S.; Luo, J.; Xie, S.-R.; Li, H.-Y.; Pu, H.-Y.; Gu, J. (2016). Robust adaptive position and force tracking control strategy for door-opening behaviour, International Journal of Simulation Modelling, Vol. 15, No. 3, 423-435, doi:10.2507/IJSIMM15(3)3.342

[21] Keymasi Khalaji, A.; Moosavian, S. A. A. (2016). Dynamic modeling and tracking control of a car with $n$ trailers, Multibody System Dynamics, Vol. 37, No. 2, 211-225, doi:10.1007/s11044-015$\underline{9472-9}$

[22] Dhaouadi, R.; Abu Hatab, A. (2013). Dynamic modelling of differential-drive mobile robots using lagrange and Newton-Euler methodologies: a unified framework, Advances in Robotics \& Automation, Vol. 2, No. 2, Paper 1000107, 7 pages, doi:10.4172/2168-9695.1000107

[23] Khalaji, A. K.; Moosavian, S. A. A. (2015). Modified transpose Jacobian control of a tractor-trailer wheeled robot, Journal of Mechanical Science and Technology, Vol. 29, No. 9, 3961-3969, doi:10.1007/s12206-015-0841-3

[24] Keymasi Khalaji, A.; Moosavian, S. A. A. (2014). Adaptive sliding mode control of a wheeled mobile robot towing a trailer, Proceedings of the Institution of Mechanical Engineers, Part I: Journal of Systems and Control Engineering, Vol. 229, No. 2, 169-183, doi:10.1177/ 0959651814550539

[25] Keymasi Khalaji, A.; Moosavian, S. A. A. (2016). Stabilization of a tractor-trailer wheeled robot, Journal of Mechanical Science and Technology, Vol. 30, No. 1, 421-428, doi:10.1007/s12206-015$\underline{1246-\mathrm{Z}}$

[26] Amezquita-Semprun, K.; del Rosario, M.; Chen, P. C. Y. (2018). Dynamics model of a differential drive mobile robot towing an off-axle trailer, International Journal of Mechanical Engineering and Robotics Research, Vol. 7, No. 6, 583-589, doi:10.18178/ijmerr.7.6.583-589

[27] Siegwart, R.; Nourbakhsh, I. R.; Scaramuzza, D. (2011). Introduction to Autonomous Mobile Robots, $2^{\text {nd }}$ edition, MIT Press, Cambridge

[28] Yavin, Y. (2007). Modelling the motion of a trolley-like car: the case where the front wheel is constraint to roll on a given plane curve, Mathematical and Computer Modelling, Vol. 46, No. 34, 307-315, doi: $10.1016 /$ j.mcm.2006.04.010 